\title{
Bounds for the solutions of unit equations
}

\author{
by \\ YAnn Bugeaud (Strasbourg) and KÁlmán Győry (Debrecen)
}

1. Introduction. Many diophantine problems can be reduced to (ordinary) unit equations and $S$-unit equations in two unknowns (for references, see e.g. [15], [24], [11], [16], [25]). Several effective bounds have been established for the heights of the solutions of such equations (see e.g. [24], [11], [25], [3] and the references given there). Except in [3], their proofs involved Baker's method and its $p$-adic analogue as well as certain quantitative results concerning independent units. The best known estimates for $S$-unit equations are due to Győry [13] and, for (ordinary) unit equations, to Schmidt [23], Sprindžuk [25] (with not completely explicit constants) and Győry [14] (with explicit constants). These led to a lot of applications.

The purpose of the present paper is to considerably improve (in completely explicit form) the above-mentioned estimates in terms of the cardinality of $S$ and of the parameters involved (degree, unit rank, regulator, class number) of the ground field. To obtain these improvements we use, among other things, some recent improvements of Waldschmidt [26] and Kunrui $\mathrm{Yu}$ [27] concerning linear forms in logarithms, some recent estimates of Brindza [5] and Hajdu [18] for fundamental systems of $S$-units, some upper and lower bounds for $S$-regulators (cf. Lemma 3 of this paper) and an idea of Schmidt [23]. Further, in our arguments we pay a particular attention to the dependence on the parameters in question. As a consequence of our result, we derive explicit bounds for the solutions of homogeneous linear equations of three terms in $S$-integers of bounded $S$-norm. These improve some earlier estimates of Györy [13], [14].

An application of our improvements is given in [17] to decomposable form equations (including Thue equations, norm form equations and discriminant form equations) in $S$-integers of a number field. Some other applications will be published in two further works.

Research of the second author was supported in part by Grant 1641 from the Hungarian National Foundation for Scientific Research and by the Foundation for Hungarian Higher Education and Research. 
2. Bounds for the solutions of $S$-unit equations. We shall use throughout this paper the following standard notation. Let $\mathbb{K}$ be an algebraic number field of degree $d$ with regulator $R_{\mathbb{K}}$, class number $h_{\mathbb{K}}$ and unit rank $r$. Denote by $O_{\mathbb{K}}$ the ring of integers of $\mathbb{K}$, and by $O_{\mathbb{K}}^{*}$ the unit group of $O_{\mathbb{K}}$. Let $S$ be a finite set of places on $\mathbb{K}$ containing the set of infinite places $S_{\infty}$. Denote by $s$ the cardinality of $S$, by $t$ the number of finite places in $S$, and by $P$ the largest of the rational primes lying below the finite places of $S$, with the convention that $P=1$ if $S=S_{\infty}$, i.e. if $t=0$. Further, denote by $O_{S}$ the ring of $S$-integers, and by $O_{S}^{*}$ the group of $S$-units in $\mathbb{K}$. Then $s-1=r+t$ is the rank of $O_{S}^{*}$. The case $s=1$ being trivial, we assume throughout the paper that $s \geq 2$. We denote by $R_{S}$ the $S$-regulator of $\mathbb{K}$ (for its definition, see Section 3). We note that for $S=S_{\infty}$ (i.e. $t=0$ ), we have $O_{S}=O_{\mathbb{K}}$ and $R_{S}=R_{\mathbb{K}}$.

For any algebraic number $\alpha$, we denote by $h(\alpha)$ the (absolute) height of $\alpha$ (cf. Section 3). There exists a $\delta_{\mathbb{K}}>0$, depending only on $\mathbb{K}$, such that $d \log h(\alpha) \geq \delta_{\mathbb{K}}$ for any $\alpha \in \mathbb{K} \backslash\{0\}$ which is not a root of unity (cf. Section 3).

Throughout this paper, we use the notation $\log ^{*} a$ for $\max \{\log a, 1\}$.

Let $\alpha, \beta$ be non-zero elements of $\mathbb{K}$ with

$$
\max \{h(\alpha), h(\beta)\} \leq H \quad(H \geq e) .
$$

Consider the $S$-unit equation

$$
\alpha x+\beta y=1 \quad \text { in } x, y \in O_{S}^{*} .
$$

When $S=S_{\infty}$ (i.e. $t=0$ ) then (1) is an (ordinary) unit equation.

TheOREM. All solutions $x, y$ of (1) satisfy

(2) $\max \{h(x), h(y)\}<\exp \left\{c_{1} P^{d} R_{S}\left(\log ^{*} R_{S}\right)\left(\log ^{*}\left(P R_{S}\right) / \log ^{*} P\right) \log H\right\}$, where

$$
c_{1}=c_{1}(d, s, \mathbb{K})=3^{25}\left(9 d^{2} / \delta_{\mathbb{K}}\right)^{s+1} s^{5 s+10} .
$$

Further, if in particular $S=S_{\infty}$ (i.e. $\left.t=0\right)$, then the bound in (2) can be replaced by

$$
\exp \left\{c_{2} R_{\mathbb{K}}\left(\log ^{*} R_{\mathbb{K}}\right) \log H\right\}
$$

where

$$
c_{2}=c_{2}(d, r, \mathbb{K})=3^{r+27}(r+1)^{5 r+17} d^{3} \delta_{\mathbb{K}}^{-(r+1)} .
$$

Remark 1. It is clear that the factor $\left(\log ^{*}\left(P R_{S}\right) / \log ^{*} P\right)$ in $(2)$ does not exceed $2 \log ^{*} R_{S}$, and if $\log ^{*} R_{S} \leq \log ^{*} P$, then it is at most 2 . Further, by Lemma 3 (cf. Section 3 ), we have

$$
R_{S} \leq R_{\mathbb{K}} h_{\mathbb{K}}\left(d \log ^{*} P\right)^{t} .
$$


Remark 2 . As is known, $R_{\mathbb{K}} h_{\mathbb{K}}$ can be estimated from above in terms of $d$ and $D_{\mathbb{K}}$, the discriminant of $\mathbb{K}$. Denote by $q$ the number of complex places of $\mathbb{K}$, and put $\Delta=(2 / \pi)^{q}\left|D_{\mathbb{K}}\right|^{1 / 2}$. If $d \geq 2$, then we have e.g. (cf. [21])

$$
R_{\mathbb{K}} h_{\mathbb{K}} \leq \Delta(\log \Delta)^{d-1-q}(d-1+\log \Delta)^{q} /(d-1) ! .
$$

Our theorem provides a considerable improvement of earlier estimates of Kotov and Trelina [19], Győry [13], [14], Schmidt [23] and Sprindžuk [25] for $S$-unit equations.

For $\alpha \in \mathbb{K} \backslash\{0\}$, the ideal generated by $\alpha$ can be uniquely written in the form $\mathfrak{a}_{1} \cdot \mathfrak{a}_{2}$ where the ideal $\mathfrak{a}_{1}$ (resp. $\mathfrak{a}_{2}$ ) is composed of prime ideals outside (resp. inside) $S$. Then the $S$-norm of $\alpha$, denoted by $N_{S}(\alpha)$, is defined as $N\left(\mathfrak{a}_{1}\right)$. In the particular case $S=S_{\infty}$, we have $N_{S_{\infty}}(\alpha)=\left|N_{\mathbb{K} / \mathbb{Q}}(\alpha)\right|$. Further, $N_{S}(\alpha)$ is a positive integer for every $\alpha \in O_{S} \backslash\{0\}$.

In some applications, it is more convenient to consider the following equation instead of (1):

$$
\begin{aligned}
& \alpha_{1} x_{1}+\alpha_{2} x_{2}+\alpha_{3} x_{3}=0 \\
& \quad \text { in } x_{i} \in O_{S} \backslash\{0\} \text { with } N_{S}\left(x_{i}\right) \leq N \text { for } i=1,2,3,
\end{aligned}
$$

where $\alpha_{1}, \alpha_{2}, \alpha_{3} \in \mathbb{K} \backslash\{0\}$ with $\max _{1 \leq i \leq 3} h\left(\alpha_{i}\right) \leq H(H \geq e)$.

Let $c_{3}=c_{3}(d, r, \mathbb{K})=r^{r+1} \delta_{\mathbb{K}}^{-(r-1)} / 2$ and let $c_{1}=c_{1}(d, s, \mathbb{K}), c_{2}=$ $c_{2}(d, r, \mathbb{K})$ denote the numbers specified in the Theorem. Then we have

Corollary. For every solution $x_{1}, x_{2}, x_{3}$ of (6) there is an $\varepsilon \in O_{S}^{*}$ such that

$$
\begin{aligned}
& \max _{1 \leq i \leq 3} h\left(\varepsilon x_{i}\right)<\exp \left\{3 c_{1} c_{3} P^{d} R_{S}\left(\log ^{*} R_{S}\right)\left(\log ^{*}\left(P R_{S}\right) / \log ^{*} P\right)\right. \\
&\left.\times\left(R_{\mathbb{K}}+t h_{\mathbb{K}} \log ^{*} P+\log (H N)\right)\right\} .
\end{aligned}
$$

Further, if $S=S_{\infty}$, then the bound in (7) can be replaced by

$$
\exp \left\{3 c_{2} c_{3} R_{\mathbb{K}}\left(\log ^{*} R_{\mathbb{K}}\right)\left(R_{\mathbb{K}}+\log (H N)\right)\right\} .
$$

Our Corollary considerably improves the earlier bounds of Györy [13], [14] concerning equation (6).

3. Bounds for $S$-units and $S$-regulators. Keeping the notations of Section 2 , denote by $M_{\mathbb{K}}$ the set of places on $\mathbb{K}$. In every place $v$ we choose a valuation $|\cdot|_{v}$ in the following way: if $v$ is infinite and corresponds to an embedding $\sigma: \mathbb{K} \rightarrow \mathbb{C}$ then we put, for every $\alpha \in \mathbb{K}$,

$$
|\alpha|_{v}=|\sigma(\alpha)|^{d_{v}}
$$

where $d_{v}=1$ or 2 according as $\sigma(\mathbb{K})$ is contained in $\mathbb{R}$ or not; if $v$ is a finite place corresponding to the prime ideal $\mathfrak{p}$ in $\mathbb{K}$ then we put $|0|_{v}=0$ and, for 
$\alpha \in \mathbb{K} \backslash\{0\}$,

$$
|\alpha|_{v}=N(\mathfrak{p})^{-\operatorname{ord}_{\mathfrak{p}}(\alpha)} .
$$
by

The (absolute) height of an algebraic number $\alpha$ contained in $\mathbb{K}$ is defined

$$
h(\alpha)=\left(\prod_{v \in M_{\mathbb{K}}} \max \left(1,|\alpha|_{v}\right)\right)^{1 / d} .
$$

This height is independent of the choice of $\mathbb{K}$. If the algebraic number $\alpha$ is of degree $n$ with minimal polynomial $a_{0}\left(X-\alpha_{1}\right) \ldots\left(X-\alpha_{n}\right) \in \mathbb{Z}[X]$ over $\mathbb{Z}$, then, by ([20], p. 54), we have

$$
h(\alpha)=\left(\left|a_{0}\right| \prod_{i=1}^{n} \max \left(1,\left|\alpha_{i}\right|\right)\right)^{1 / n} .
$$

There is a positive constant $\delta_{\mathbb{K}}$, depending only on $\mathbb{K}$, such that for every non-zero algebraic number $\alpha \in \mathbb{K}$ which is not a root of unity we have $\log h(\alpha) \geq \delta_{\mathbb{K}} / d$ (we recall that $d$ denotes the degree of $\mathbb{K}$ ). Further, if $\alpha$ is not an algebraic integer then (8) implies that $\log h(\alpha) \geq \log 2 / d$. Hence we have $\delta_{\mathbb{K}} \leq \log 2$.

It is easy to see that we can take

$$
\delta_{\mathbb{K}}=\frac{\log 2}{r+1} \quad \text { for } d=1,2,
$$

where $r$ denotes the unit rank of $\mathbb{K}$. Further, it follows from results of Blanksby and Montgomery [2] and of Dobrowolski [7], [8] that both

$$
\delta_{\mathbb{K}}=\frac{1}{53 d \log 6 d} \quad \text { and } \quad \delta_{\mathbb{K}}=\frac{1}{1201}\left(\frac{\log \log d}{\log d}\right)^{3}\left({ }^{1}\right)
$$

are appropriate choices for $d \geq 3$. For large $d$, the factor $1 / 1201$ can be replaced by a larger one (see e.g. [9]).

We recall that $s$ denotes the cardinality of $S$. For $v \in S$, denote by $|\cdot|_{v}$ the corresponding valuation normalized as above. Let $v_{1}, \ldots, v_{s-1}$ be a subset of $S$, and let $\left\{\varepsilon_{1}, \ldots, \varepsilon_{s-1}\right\}$ be a fundamental system of $S$-units in $\mathbb{K}$. Denote by $R_{S}$ the absolute value of the determinant of the matrix $\left(\log \left|\varepsilon_{i}\right|_{v_{j}}\right)_{i, j=1, \ldots, s-1}$. It is easy to verify that $R_{S}$ is a positive number which is independent of the choice of $v_{1}, \ldots, v_{s-1}$ and of the fundamental system of $S$-units $\left\{\varepsilon_{1}, \ldots, \varepsilon_{s-1}\right\} . R_{S}$ is called the $S$-regulator of $\mathbb{K}$. If in particular $S=S_{\infty}$, then we have $R_{S}=R_{\mathbb{K}}$.

There are several quantitative results in the literature for units and $S$ units of small height; for references, see e.g. [24], [5] and [18]. The following lemma is in fact due to Hajdu [18]. It is an extended version of an earlier

$\left({ }^{1}\right)$ Added in proof. By a recent result of P. M. Voutier (see this issue), one can take here $1 / 4$ instead of $1 / 1201$. 
theorem of Brindza [5]. For convenience of the reader, we give here a proof for Lemma 1 with a slightly better value for $c_{4}$ than in [18].

Put

$$
c_{4}=c_{4}(d, s)=((s-1) !)^{2} /\left(2^{s-2} d^{s-1}\right)
$$

and

$$
c_{5}=c_{5}(d, s, \mathbb{K})=c_{4}\left(\frac{\delta_{\mathbb{K}}}{d}\right)^{2-s}, \quad c_{6}=c_{6}(d, s, \mathbb{K})=c_{4} d^{s-1} \delta_{\mathbb{K}}^{-1} .
$$

Lemma 1. There exists in $\mathbb{K}$ a fundamental system $\left\{\varepsilon_{1}, \ldots, \varepsilon_{s-1}\right\}$ of $S$-units with the following properties:

$$
\begin{gathered}
\prod_{i=1}^{s-1} \log h\left(\varepsilon_{i}\right) \leq c_{4} R_{S} ; \\
\log h\left(\varepsilon_{i}\right) \leq c_{5} R_{S}, \quad i=1, \ldots, s-1 ;
\end{gathered}
$$

(iii) the absolute values of the entries of the inverse matrix of $\left(\log \left|\varepsilon_{i}\right|_{v_{j}}\right)_{i, j=1, \ldots, s-1}$ do not exceed $c_{6}$.

Proof. We shall combine some arguments from the proofs of [5] and [18]. For $\alpha \in \mathbb{K} \backslash\{0\}$ put

$$
\mathbf{v}(\alpha)=\left(\log |\alpha|_{v_{1}}, \ldots, \log |\alpha|_{v_{s-1}}\right) .
$$

The lattice $\Lambda$ in $\mathbb{R}^{s-1}$ spanned by the vectors $\mathbf{v}(\eta)$ with $\eta \in O_{S}^{*}$ has determinant $R_{S}$.

The function $F: \mathbb{R}^{s-1} \rightarrow \mathbb{R}$ defined by

$$
F(\mathbf{x})=\left|x_{1}\right|+\ldots+\left|x_{s-1}\right|
$$

for $\mathbf{x}=\left(x_{1}, \ldots, x_{s-1}\right) \in \mathbb{R}^{s-1}$ is a symmetric convex distance function (cf. [6], Ch. IV), i.e. it is non-negative, continuous, $F(\alpha \mathbf{x})=\alpha F(\mathbf{x})(\alpha \geq 0$ real $)$ and $F(\mathbf{x}+\mathbf{y}) \leq F(\mathbf{x})+F(\mathbf{y})$ for $\mathbf{x}, \mathbf{y} \in \mathbb{R}^{s-1}$. Denote by $V_{F}$ the volume of the bounded set $\left\{\mathbf{x} \in \mathbb{R}^{s-1} \mid F(\mathbf{x})<1\right\}$. It is easy to check that $V_{F}=$ $2^{s-1} /(s-1)$ !. By a theorem of Minkowski (cf. [6], Ch. VIII) the successive minima $\lambda_{1}, \ldots, \lambda_{s-1}$ of $\Lambda$ with respect to $F$ have the property

$$
\lambda_{1} \ldots \lambda_{s-1} \leq 2^{s-1} R_{S} / V_{F}=(s-1) ! R_{S} .
$$

Further, there are multiplicatively independent $S$-units $\eta_{1}, \ldots, \eta_{s-1}$ for which

$$
F\left(\mathbf{v}\left(\eta_{i}\right)\right)=\lambda_{i}, \quad i=1, \ldots, s-1 .
$$

It follows (cf. [6], p. 135, Lemma 8) that there exists a fundamental system $\left\{\varepsilon_{1}, \ldots, \varepsilon_{s-1}\right\}$ of $S$-units such that

$$
F\left(\mathbf{v}\left(\varepsilon_{i}\right)\right) \leq \max \{1, i / 2\} F\left(\mathbf{v}\left(\eta_{i}\right)\right), \quad i=1, \ldots, s-1 .
$$


However, for every $\eta \in O_{S}^{*}$, we have $\prod_{v \in S}|\eta|_{v}=1$, hence

$$
\log h(\eta)=\frac{1}{d} \sum_{v \in S} \max \left\{0, \log |\eta|_{v}\right\}=\left.\frac{1}{2 d} \sum_{v \in S}|\log | \eta\right|_{v} \mid
$$

which implies that

$$
\frac{1}{2 d} F(\mathbf{v}(\eta)) \leq \log h(\eta) \leq \frac{1}{d} F(\mathbf{v}(\eta)) .
$$

Hence, by (12), (11), (10) and (9), we have

$$
\begin{aligned}
\prod_{i=1}^{s-1} \log h\left(\varepsilon_{i}\right) & \leq \frac{1}{d^{s-1}} \prod_{i=1}^{s-1} F\left(\mathbf{v}\left(\varepsilon_{i}\right)\right) \leq \frac{(s-1) !}{2^{s-2} d^{s-1}} \prod_{i=1}^{s-1} F\left(\mathbf{v}\left(\eta_{i}\right)\right) \\
& \leq((s-1) !)^{2} R_{S} /\left(2^{s-2} d^{s-1}\right)
\end{aligned}
$$

which proves (i).

(ii) follows immediately from (i) and $\log h\left(\varepsilon_{i}\right) \geq \delta_{\mathbb{K}} / d$ for $i=1, \ldots, s-1$.

To prove (iii), let $E=\left(\log \left|\varepsilon_{i}\right|_{v_{j}}\right)_{i, j=1, \ldots, s-1}$ and $e_{i j}=\operatorname{det}\left(E_{i j}\right) / \operatorname{det}(E)$, where $E_{i j}$ denotes the matrix obtained from $E$ by omitting the $i$ th row and $j$ th column. It follows from (13) and Hadamard's inequality that

$$
\left|\operatorname{det}\left(E_{i j}\right)\right| \leq \prod_{\substack{p=1 \\ p \neq i}}^{s-1} \sqrt{\sum_{\substack{q=1 \\ q \neq j}}^{s-1}\left(\log \left|\varepsilon_{p}\right|_{v_{q}}\right)^{2}} \leq \prod_{\substack{p=1 \\ p \neq i}}^{s-1} F\left(\mathbf{v}\left(\varepsilon_{p}\right)\right) \leq c_{4} R_{S} / F\left(\mathbf{v}\left(\varepsilon_{i}\right)\right) .
$$

Together with (12), $|\operatorname{det}(E)|=R_{S}$ and $\log h\left(\varepsilon_{i}\right) \geq \delta_{\mathbb{K}} / d$ this implies $\left|e_{i j}\right| \leq$ $c_{4} \delta_{\mathbb{K}}^{-1} d^{s-1}$, which completes the proof.

The next lemma has various versions in the literature (for references, see e.g. [15], [24], [10], [18]). Our lemma is an explicit version of Lemma 10 of $[10]$.

Let $c_{3}=c_{3}(d, r, \mathbb{K})$ denote the constant specified in the Corollary.

Lemma 2. For every $\alpha \in O_{S} \backslash\{0\}$ and every integer $n \geq 1$ there exists an $S$-unit \& such that

$$
h\left(\varepsilon^{n} \alpha\right) \leq N_{S}(\alpha)^{1 / d} \exp \left\{n\left(c_{3} R_{\mathbb{K}}+t h_{\mathbb{K}} \log ^{*} P\right)\right\} .
$$

Pr o o f. First consider the case when $S=S_{\infty}$. So let $\alpha \in O_{\mathbb{K}} \backslash\{0\}$ and put $M=\left|N_{\mathbb{K} / \mathbb{Q}}(\alpha)\right|$. Let $S_{\infty}=\left\{v_{1}, \ldots, v_{r+1}\right\}$ and $L(\alpha)=\left.\max _{1 \leq i \leq r}|\log | \alpha\right|_{v_{i}} \mid$. Then there are multiplicatively independent units $\eta_{1}, \ldots, \eta_{r}$ in $O_{\mathbb{K}}$ such that $L\left(\eta_{1}\right) \ldots L\left(\eta_{r}\right) \leq R_{\mathbb{K}}$ (cf. [14]). On the other hand, we have $L\left(\eta_{j}\right) \geq$ $(d / r) \log h\left(\eta_{j}\right) \geq \delta_{\mathbb{K}} / r$, whence $L\left(\eta_{j}\right) \leq r^{r-1} \delta_{\mathbb{K}}^{-(r-1)} R_{\mathbb{K}}$ for each $j$.

Consider the system of linear equations

$$
\sum_{j=1}^{r} X_{j} \log \left|\eta_{j}\right|_{v_{i}}=-\log \left(M^{-d_{v_{i}} / d}|\alpha|_{v_{i}}\right), \quad i=1, \ldots, r+1,
$$


in $X_{1}, \ldots, X_{r}$. It has a unique solution $x_{1}, \ldots, x_{r}$ in $\mathbb{R}$. For $1 \leq j \leq r$, there exist $b_{j} \in \mathbb{Z}$ and $\varrho_{j} \in \mathbb{R}$ with $\left|\varrho_{j}\right| \leq n / 2$ such that $x_{j}=n b_{j}+\varrho_{j}$. Putting $\eta_{1}^{b_{1}} \ldots \eta_{r}^{b_{r}}=\varepsilon$, we infer that

$$
\begin{aligned}
\left|\log \left(M^{-d_{v_{i}} / d}\left|\alpha \varepsilon^{n}\right|_{v_{i}}\right)\right| & =\left.\left|\sum_{j=1}^{r} \varrho_{j} \log \right| \eta_{j}\right|_{v_{i}} \mid \\
& \leq\left.\frac{n r}{2} \max _{1 \leq j \leq r}|\log | \eta_{j}\right|_{v_{i}} \mid \leq \frac{n r}{2} \cdot r \max _{1 \leq j \leq r} L\left(\eta_{j}\right) \\
& \leq n c_{3} R_{\mathbb{K}}, \quad i=1, \ldots, r+1,
\end{aligned}
$$

which implies (14).

The general case of our lemma follows from the case $S=S_{\infty}$ as in the proof of Lemma 10 of [10].

Denote by $\mathfrak{p}_{1}, \ldots, \mathfrak{p}_{t}$ the prime ideals in $\mathbb{K}$ corresponding to the finite places in $S$. We recall that $P$ denotes the largest of the rational primes lying below of these prime ideals.

The following lemma is an improvement of some estimates of Pethő [22] and Hajdu [18] for $R_{S}$. It should, however, be remarked that Pethő's estimate was established in a more general situation, for some $S$-orders instead of $O_{S}$.

LEMMA 3. If $t>0$, then

$$
R_{S} \leq R_{\mathbb{K}} h_{\mathbb{K}} \prod_{i=1}^{t} \log N\left(\mathfrak{p}_{i}\right) \leq R_{\mathbb{K}} h_{\mathbb{K}}\left(d \log ^{*} P\right)^{t}
$$

and

$$
R_{S} \geq R_{\mathbb{K}} \prod_{i=1}^{t} \log N\left(\mathfrak{p}_{i}\right) \geq c_{7}(\log 2)\left(\log ^{*} P\right),
$$

where $c_{7}=0.2052$.

Proof. $O_{S}^{*} / O_{\mathbb{K}}^{*}$ is a free abelian group of rank $t$ which is isomorphic to the multiplicative group of principal ideals in $\mathbb{K}$ generated by the elements of $O_{S}^{*}$. This latter group is a subgroup of finite index, say $i_{S}$, of the multiplicative group generated by $\mathfrak{p}_{1}, \ldots, \mathfrak{p}_{t}$ and we have $i_{S} \leq h_{\mathbb{K}}$. Hence, as is known (see e.g. [4], pp. 85 and 125), this subgroup has a basis of the form

$$
\left(\varepsilon_{i}\right)=\mathfrak{p}_{i}^{a_{i i}} \mathfrak{p}_{i+1}^{a_{i, i+1}} \ldots \mathfrak{p}_{t}^{a_{i t}}, \quad i=1, \ldots, t,
$$

with rational integers $a_{i j}$ such that $a_{i i}>0$ for $i=1, \ldots, t$ and that $a_{11} \ldots a_{t t}=i_{S}$. It now follows that if $\left\{\varepsilon_{t+1}, \ldots, \varepsilon_{t+r}\right\}$ is a fundamental system of units in $O_{\mathbb{K}}$ then $\left\{\varepsilon_{1}, \ldots, \varepsilon_{t}, \ldots, \varepsilon_{t+r}\right\}$ is a fundamental system of $S$-units in $\mathbb{K}$. Consequently, it is easy to see that

$$
R_{S}=\left|\operatorname{det}\left(\log \left|\varepsilon_{i}\right|_{v_{j}}\right)_{i, j=1, \ldots, r+t}\right|=R_{\mathbb{K}} i_{S} \prod_{i=1}^{t} \log N\left(\mathfrak{p}_{i}\right),
$$


which gives (16). Inequalities (17) follow from (18) and the estimate $R_{\mathbb{K}} \geq c_{7}$ of Friedman [12].

We remark that, in our Theorem and its Corollary, the improvements of the previous bounds in terms of $R_{\mathbb{K}}, h_{\mathbb{K}}$ and $P$ are mainly due to the use of fundamental systems of $S$-units, $S$-regulators as well as Lemmas 1 to 3 .

4. Estimates for linear forms in logarithms. In our proofs, we shall use the best known estimates, due to Waldschmidt [26] and Kunrui Yu [27] respectively, for linear forms in logarithms in the complex and in the $p$-adic case. We shall formulate them in a more convenient form for our purpose. These estimates enable us to considerably improve the previous bounds for the solutions of equation (1) in terms of $d, r$ and $s$.

Let $\alpha_{1}, \ldots, \alpha_{n}(n \geq 2)$ be non-zero algebraic numbers and let $\mathbb{K}=$ $\mathbb{Q}\left(\alpha_{1}, \ldots, \alpha_{n}\right)$. Put $[\mathbb{K}: \mathbb{Q}]=d$. Let $A_{1}, \ldots, A_{n}$ be real numbers such that

$$
\log A_{i} \geq \max \left\{\log h\left(\alpha_{i}\right), \frac{\left|\log \alpha_{i}\right|}{3.3 d}, \frac{1}{d}\right\}, \quad i=1, \ldots, n,
$$

where $\log$ denotes the principal value of the logarithm. Let $b_{1}, \ldots, b_{n}$ be rational integers and put $B=\max \left\{\left|b_{1}\right|, \ldots,\left|b_{n}\right|, 3\right\}$. Further, set

$$
\Lambda=\alpha_{1}^{b_{1}} \ldots \alpha_{n}^{b_{n}}-1 \text {. }
$$

In Proposition 1, it will be convenient to make the following technical assumptions:

$$
B \geq \log A_{n} \exp \{4(n+1)(7+3 \log (n+1))\}
$$

and

$$
7+3 \log (n+1) \geq \log d .
$$

Proposition 1 is a consequence of Corollary 10.1 of Waldschmidt [26].

Proposition 1 (M. Waldschmidt [26]). If $\Lambda \neq 0, b_{n}=1$ and (20), (21) hold, then

$$
|\Lambda| \geq \exp \left\{-c_{8}(n) d^{n+2} \log A_{1} \ldots \log A_{n} \log \left(\frac{2 n B}{\log A_{n}}\right)\right\},
$$

where $c_{8}(n)=1500 \cdot 38^{n+1}(n+1)^{3 n+9}$.

We remark that a recent explicit estimate of Baker and Wüstholz [1] for linear forms in logarithms would give here a smaller value for $c_{8}(n)$ in terms of $n$. However, the lower bound in (22) is better in terms of $A_{n}$, which is essential for our present applications.

Proof of Proposition 1 . We denote by $\log$ the principal value of the logarithm. Setting $\alpha_{0}=-1$, there is a $b_{0} \in \mathbb{Z}$ such that $\left|b_{0}\right| \leq$ 
$\left|b_{1}\right|+\ldots+\left|b_{n-1}\right|+2 \leq n B$ and that

$$
\log \left(\alpha_{1}^{b_{1}} \ldots \alpha_{n}^{b_{n}}\right)=\sum_{j=1}^{n} b_{j} \log \alpha_{j}+b_{0} \log \alpha_{0}:=\Omega,
$$

where $b_{n}=1$. It suffices to deal with the case when $|\Lambda| \leq 1 / 3$. Since $|\log z| \leq$ $2|z-1|$ for any $z \in \mathbb{C}$ with $|z-1| \leq 1 / 3$, we get

$$
|\Lambda| \geq|\Omega| / 2 \text {. }
$$

After some calculations and under the conditions (20), (21), Corollary 10.1 of [26] implies the following inequality with the choice $E=e, f=1 /(3.3 d)$ and $g=2$ :

$$
|\Omega| \geq 2 \exp \left\{-c_{8}(n) d^{n+2} \log A_{1} \ldots \log A_{n} \log \left(\frac{2 n B}{\log A_{n}}\right)\right\} .
$$

Together with (23) this implies (22).

In Proposition 2 , let $v=v_{\mathfrak{p}}$ be a finite place on $\mathbb{K}$, corresponding to the prime ideal $\mathfrak{p}$ of $\mathbb{K}$. Let $p$ denote the rational prime lying below $\mathfrak{p}$, and denote by $|\cdot|_{v}$ the non-archimedean valuation normalized as in Section 3. Instead of (19), assume now that $A_{1}, \ldots, A_{n}$ are real numbers such that

$$
\log A_{i} \geq \max \left\{\log h\left(\alpha_{i}\right),\left|\log \alpha_{i}\right| /(10 d), \log p\right\}, \quad i=1, \ldots, n .
$$

The following proposition is a simple consequence of the main result of Kunrui $\mathrm{Yu}[27]$.

Proposition 2 (Kunrui Yu [27]). Let

$$
\Phi=c_{9}(n)(d / \sqrt{\log p})^{2(n+1)} p^{d} \log A_{1} \ldots \log A_{n} \log (10 n d \log A),
$$

where $c_{9}(n)=22000(9.5(n+1))^{2(n+1)}$ and $A=\max \left\{A_{1}, \ldots, A_{n}, e\right\}$. If $\Lambda \neq 0$ then

$$
|\Lambda|_{v} \geq \exp \{-d(\log p) \Phi \log (d B)\} .
$$

Further, if $b_{n}=1$ and $A_{n} \geq A_{i}$ for $i=1, \ldots, n-1$, then $A$ can be replaced by $\max \left\{A_{1}, \ldots, A_{n-1}, e\right\}$ and for any $\delta$ with $0<\delta \leq 1$, we have

$$
|\Lambda|_{v} \geq \exp \left\{-d(\log p) \max \left\{\Phi \log \left(\delta^{-1} \Phi / \log A_{n}\right), \delta B\right\}\right\} .
$$

Proof. This is a reformulation of the result presented in the introduction of Kunrui $\mathrm{Yu}$ [27].

Remark 6. We remark that, in Propositions 1 and 2, the condition $\mathbb{K}=$ $\mathbb{Q}\left(\alpha_{1}, \ldots, \alpha_{n}\right)$ can be removed. It is enough to assume that $\mathbb{K}$ is an algebraic number field of degree $d$ which contains $\alpha_{1}, \ldots, \alpha_{n}$. This observation will be needed in Section 5. 
5. Proofs of the Theorem and the Corollary of

Proof of the Theorem. Let $x, y$ be an arbitrary but fixed solution

$$
\alpha x+\beta y=1 \quad \text { in } x, y \in O_{S}^{*} .
$$

We assume that $h(x) \geq h(y)$. Let $\left\{\varepsilon_{1}, \ldots, \varepsilon_{s-1}\right\}$ be a fundamental system of $S$-units in $\mathbb{K}$ with the properties specified in Lemma 1 . Then we can write

$$
y=\zeta \varepsilon_{1}^{b_{1}} \ldots \varepsilon_{s-1}^{b_{s-1}}
$$

with a root of unity $\zeta$ in $\mathbb{K}$ and with rational integers $b_{1}, \ldots, b_{s-1}$. Put $B=\max \left\{\left|b_{1}\right|, \ldots,\left|b_{s-1}\right|, 3\right\}$ and $S=\left\{v_{1}, \ldots, v_{s}\right\}$. Then (25) implies

$$
\log |y|_{v_{j}}=\sum_{i=1}^{s-1} b_{i} \log \left|\varepsilon_{i}\right|_{v_{j}}, \quad j=1, \ldots, s-1,
$$

whence, by (iii) of Lemma 1 and (12), we get

$$
B \leq\left. c_{6} \sum_{j=1}^{s-1}|\log | y\right|_{v_{j}} \mid \leq 2 d c_{6} \log h(y) \leq 2 d c_{6} \log h(x)
$$

with the $c_{6}=c_{6}(d, s, \mathbb{K})$ specified in Lemma 1 .

Let $v \in S$ for which $|x|_{v}$ is minimal. Setting $\alpha_{s}=\zeta \beta$ and $b_{s}=1$, we deduce from (1) that

$$
|\alpha x|_{v}=\left|\varepsilon_{1}^{b_{1}} \ldots \varepsilon_{s-1}^{b_{s-1}} \alpha_{s}^{b_{s}}-1\right|_{v} .
$$

We shall derive a lower bound for $|\alpha x|_{v}$.

First assume that $v$ is infinite. In order to apply Proposition 1, put

$$
\begin{aligned}
\log A_{i} & =\delta_{\mathbb{K}}^{-1} \log h\left(\varepsilon_{i}\right), \quad i=1, \ldots, s-1, \\
\log A_{s} & =\delta_{\mathbb{K}}^{-1} \log H .
\end{aligned}
$$

It is easy to check that $7+3 \log (s+1) \geq \log d$. Further, we may assume that

$$
B \geq \log A_{s} \exp \{4(s+1)(7+3 \log (s+1))\} .
$$

Indeed, (1) implies that

$$
h(x) \leq 2 H^{2} h(y) .
$$

Further, it follows from (25) and (ii) of Lemma 1 that

$$
h(y) \leq \prod_{i=1}^{s-1} h\left(\varepsilon_{i}\right)^{\left|b_{i}\right|} \leq \exp \left\{(s-1) c_{5} R_{S} B\right\} .
$$

Hence, if (29) does not hold, we get at once a bound for $h(x)$ which is better than that in the Theorem. 
We have $|\cdot|_{v}=|\sigma(\cdot)|^{d_{v}}$ for some $\sigma: \mathbb{K} \rightarrow \mathbb{C}$. Applying $\sigma$ to equation (1) and then omitting $\sigma$ everywhere, we may assume that $|\cdot|_{v}=|\cdot|^{d_{v}}$. On applying now Proposition 1 to (27) and using (i) of Lemma 1, we derive that

$$
|\alpha x|_{v} \geq \exp \left\{-c_{10} R_{S} \log H \log \left(\frac{c_{11} B}{\log H}\right)\right\},
$$

where $c_{10}=d_{v} c_{8}(s) c_{4} d^{s+2} \delta_{\mathbb{K}}^{-s}$ and $c_{11}=2 s \delta_{\mathbb{K}}$.

Since $|x|_{v}$ is minimal, we have

$$
h(x)=h(1 / x) \leq|x|_{v}^{-(s-1) / d} .
$$

Hence it follows from (32), (26) and $|\alpha|_{v} \leq H^{d}$ that

$$
\frac{\log h(x)}{\log H} \leq \frac{2(s-1)}{d} c_{10} R_{S} \log \left(\frac{c_{12} \log h(x)}{\log H}\right)
$$

where $c_{12}=2 d c_{6} c_{11}$. This gives $\left({ }^{2}\right)$

$$
h(x) \leq \exp \left\{c_{13} R_{S}\left(\log ^{*} R_{S}\right) \log H\right\}
$$

with

$$
c_{13}=3^{s+26} d^{3} \delta_{\mathbb{K}}^{-s} s^{5 s+12} .
$$

We remark that in the particular case $S=S_{\infty}$, i.e. when $t=0,(34)$ implies the second part of the Theorem.

Next assume that $v$ is finite. To apply Proposition 2, we put now

$$
\begin{aligned}
\log A_{i} & =\delta_{\mathbb{K}}^{-1} \log h\left(\varepsilon_{i}\right)+\log ^{*} P, \quad i=1, \ldots, s-1, \\
\log A_{s} & =\delta_{\mathbb{K}}^{-1} \log H+\log ^{*} P .
\end{aligned}
$$

Using (i) of Lemma 1, we get

$\log A_{1} \ldots \log A_{s-1}$

$$
\begin{aligned}
\leq & \prod_{i=1}^{s-1}\left(\delta_{\mathbb{K}}^{-1} \log h\left(\varepsilon_{i}\right)\right)\left(\sum_{j=0}^{s-1}\left(\begin{array}{c}
s-1 \\
j
\end{array}\right)\left(d \log ^{*} P\right)^{j}-\left(d \log ^{*} P\right)^{s-1}\right) \\
& +\left(\log ^{*} P\right)^{s-1} \\
\leq & \left(\log ^{*} P\right)^{s-2}\left(c_{14} R_{S}+\log ^{*} P\right)
\end{aligned}
$$

with $c_{14}=(s / d)((s-1) !)^{2} \delta_{\mathbb{K}}^{-(s-1)}$. Together with the second inequality of Lemma 3 this gives

$$
\log A_{1} \ldots \log A_{s-1} \leq 2 c_{14} R_{S}\left(\log ^{*} P\right)^{s-2} .
$$

$\left({ }^{2}\right)$ In certain applications (e.g. in case of practical solutions of $S$-unit equations), it can be more useful to work with our upper bounds of $B$, provided by (26), (34) and (43). 
We distinguish two cases. First assume that $\log H<c_{5} R_{S}$. Then, by Lemmas 1 and 3, we have

$$
\log A:=\max _{1 \leq i \leq s} \log A_{i} \leq c_{15} R_{S}
$$

with $c_{15}=c_{5} \delta_{\mathbb{K}}^{-1}+\left(c_{7} \log 2\right)^{-1}$. We now apply to (27) the first part of Proposition 2. Putting

$$
\Phi=c_{16} \frac{P^{d}}{\left(\log ^{*} P\right)^{s+1}} \log A_{1} \ldots \log A_{s} \log (10 s d \log A)
$$

with $c_{16}=c_{9}(s)\left(d^{2} / \log 2\right)^{s+1}$, we infer that

$$
|\alpha x|_{v} \geq \exp \left\{-d\left(\log ^{*} P\right) \Phi \log (d B)\right\},
$$

whence, by (33), (26) and $|\alpha|_{v} \leq H^{d}$,

$$
\log h(x) \leq 2(s-1)\left(\log ^{*} P\right) \Phi \log \left(c_{17} \log h(x)\right)
$$

follows with $c_{17}=2 d^{2} c_{6}$. Together with (36), (37) and $\log H<c_{5} R_{S}$ this gives

$$
h(x) \leq \exp \left\{c_{18} P^{d} R_{S}\left(\log ^{*} R_{S}\right)\left(\log ^{*}\left(P R_{S}\right) / \log ^{*} P\right) \log H\right\},
$$

where

$$
c_{18}=3^{26}\left(18 d^{2} / \delta_{\mathbb{K}}\right)^{s+1} s^{4 s+7} .
$$

Next assume that $\log H \geq c_{5} R_{S}$. Then, by Lemmas 1 and 3 , we have $A_{s} \geq A_{i}$ for $i=1, \ldots, s-1$ and

$$
\log A:=\max _{1 \leq i \leq s-1} \log A_{i} \leq c_{15} R_{S} .
$$

Consider now the above defined $\Phi$ with this value of $\log A$. First we give an upper bound for $h(x)$ in terms of $\Phi$.

If $B<\Phi\left(\log ^{*} P\right) /\left(c_{5} R_{S}\right)$ then (30), (31) and (35) imply that

$$
h(x) \leq 2 H^{2} \exp \left\{(s-1) \Phi \log ^{*} P\right\}<\exp \left\{s \Phi \log ^{*} P\right\} .
$$

Assume now that $B \geq \Phi\left(\log ^{*} P\right) /\left(c_{5} R_{S}\right)$. We apply the second part of Proposition 2 to (27). Putting $\delta=\Phi\left(\log ^{*} P\right) /\left(B c_{5} R_{S}\right)$ we obtain

$$
|\alpha x|_{v} \geq \exp \left\{-d\left(\log ^{*} P\right) \Phi \log \left(\frac{B c_{5} R_{S}}{\log ^{*} P \log A_{s}}\right)\right\} .
$$

Hence, proceeding again as above, we deduce that

$$
\frac{\log h(x)}{\log ^{*} P \log A_{s}} \leq 2(s-1)\left(\Phi / \log A_{s}\right) \log \left(\frac{c_{19} R_{S} \log h(x)}{\log ^{*} P \log A_{s}}\right)
$$

with $c_{19}=2 d c_{6} c_{5}$. From this we infer as above that

$$
h(x) \leq \exp \left\{c_{20} \Phi\left(\log ^{*} P\right) \log ^{*}\left(P R_{S}\right)\right\},
$$

where $c_{20}=19(s-1) \log \left(c_{16}\right)$. 
The right hand side of (42) is greater than that of (41). Lemma 3, (35) and $\log H \geq c_{5} R_{S}$ imply that $\log A_{s}<c_{21} \log H$ with $c_{21}=\left(c_{5} c_{7} \log 2\right)^{-1}+$ $\delta_{\mathbb{K}}^{-1}$. Hence, estimating from above $\Phi$, we obtain in both cases that

$$
h(x) \leq \exp \left\{c_{18} P^{d} R_{S}\left(\log ^{*} R_{S}\right)\left(\log ^{*}\left(P R_{S}\right) / \log ^{*} P\right) \log H\right\},
$$

with the constant $c_{18}$ defined above. However, it is easy to verify that both $c_{13}$ in (34) and $c_{18}$ in (39) and (43) are less than $c_{1}=c_{1}(d, s, \mathbb{K})$ specified in the Theorem. This completes the proof of our assertion.

Proof of the Corollary. Let $x_{1}, x_{2}, x_{3}$ be a solution of (6). Then, by Lemma 2, there are $\varepsilon_{i} \in O_{S}^{*}$ such that

$$
h\left(\varepsilon_{i} x_{i}\right) \leq N^{1 / d} \exp \left\{c_{3} R_{\mathbb{K}}+t h_{\mathbb{K}} \log ^{*} P\right\}
$$

with the constant $c_{3}$ specified in Lemma 2. Put

$$
\alpha=\frac{\alpha_{1}\left(\varepsilon_{1} x_{1}\right)}{\alpha_{3}\left(\varepsilon_{3} x_{3}\right)}, \quad \beta=\frac{\alpha_{2}\left(\varepsilon_{2} x_{2}\right)}{\alpha_{3}\left(\varepsilon_{3} x_{3}\right)} .
$$

Then $x=-\varepsilon_{3} / \varepsilon_{1}, y=-\varepsilon_{3} / \varepsilon_{2}$ is a solution of equation (1).

We have

$$
\max \{h(\alpha), h(\beta)\} \leq \exp \left\{2 c_{3}\left(R_{\mathbb{K}}+t h_{\mathbb{K}} \log ^{*} P+\log (H N)\right)\right\} .
$$

Now our Theorem provides an explicit upper bound for $\max \{h(x), h(y)\}$. Together with (44), this implies (7) with the choice $\varepsilon=-\varepsilon_{3}$.

Acknowledgements. Most of the arguments used in the present paper were found independently by the two authors. The first named author would like to thank Professor Maurice Mignotte for his constant encouragement.

\section{References}

[1] A. Baker and G. Wüstholz, Logarithmic forms and group varieties, J. Reine Angew. Math. 442 (1993), 19-62.

[2] P. E. Blanksby and H. L. Montgomery, Algebraic integers near the unit circle, Acta Arith. 18 (1971), 355-369.

[3] E. Bombieri, Effective diophantine approximation on $G_{m}$, Ann. Scuola Norm. Sup. Pisa (IV) 20 (1993), 61-89.

[4] Z. I. Borevich and I. R. Shafarevich, Number Theory, 2nd ed., Academic Press, New York, 1967.

[5] B. Brindza, On the generators of $S$-unit groups in algebraic number fields, Bull. Austral. Math. Soc. 43 (1991), 325-329.

[6] J. W. S. Cassels, An Introduction to the Geometry of Numbers, Grundlehren Math. Wiss. 99, Springer, Berlin, 1959.

[7] E. Dobrowolski, On the maximal modulus of conjugates of an algebraic integer, Bull. Acad. Polon. Sci. 26 (1978), 291-292.

[8] - On a question of Lehmer and the number of irreducible factors of a polynomial, Acta Arith. 34 (1979), 391-401. 
[9] A. Dubickas, On a conjecture of A. Schinzel and H. Zassenhaus, ibid. 63 (1993), $15-20$.

[10] J. H. Evertse and K. Györy, Effective finiteness results for binary forms with given discriminant, Compositio Math. 79 (1991), 169-204.

[11] J. H. Evertse, K. Győry, C. L. Stewart and R. Tijdeman, S-unit equations and their applications, in: New Advances in Transcendence Theory, A. Baker (ed.), Cambridge University Press, 1988, 110-174.

[12] E. Friedman, Analytic formulas for regulators of number fields, Invent. Math. 98 (1989), 599-622.

[13] K. Győry, On the number of solutions of linear equations in units of an algebraic number field, Comment. Math. Helv. 54 (1979), 583-600.

[14] - On the solutions of linear diophantine equations in algebraic integers of bounded norm, Ann. Univ. Sci. Budapest. Eötvös Sect. Math. 22/23 (1980), 225-233.

[15] - Résultats effectifs sur la représentation des entiers par des formes décomposables, Queen's Papers in Pure and Appl. Math. 56 (1980).

[16] —, Some recent applications of $S$-unit equations, in: Journées Arithmétiques de Genève 1991, D. F. Coray and Y.-F. S. Pétermann (eds.), Astérisque 209 (1992), $17-38$.

[17] - Bounds for the solutions of decomposable form equations, to appear.

[18] L. Hajdu, A quantitative version of Dirichlet's $S$-unit theorem in algebraic number fields, Publ. Math. Debrecen 42 (1993), 239-246.

[19] S. V. Kotov und L. A. Trelina, S-ganze Punkte auf elliptischen Kurven, J. Reine Angew. Math. 306 (1979), 28-41.

[20] S. Lang, Fundamentals of Diophantine Geometry, Springer, Berlin, 1983.

[21] H. W. Lenstra, Jr., Algorithms in algebraic number theory, Bull. Amer. Math. Soc. 26 (1992), 211-244.

[22] A. Pethő, Beiträge zur Theorie der S-Ordnungen, Acta Math. Acad. Sci. Hungar. 37 (1981), 51-57.

[23] W. M. Schmidt, Integer points on curves of genus 1, Compositio Math. 81 (1992), $33-59$.

[24] T. N. Shorey and R. Tijdeman, Exponential Diophantine Equations, Cambridge University Press, Cambridge, 1986.

[25] V. G. Sprindžuk, Classical Diophantine Equations, Lecture Notes in Math. 1559, Springer, 1993.

[26] M. Waldschmidt, Minorations de combinaisons linéaires de logarithmes de nombres algébriques, Canad. J. Math. 45 (1993), 176-224.

[27] K. Y u, Linear forms in p-adic logarithms III, Compositio Math. 91 (1994), 241-276.

U. F. R. DE MATHÉMATIQUES

UNIVERSITÉ LOUIS PASTEUR

7, RUE RENÉ DESCARTES

67084 STRASBOURG, FRANCE

E-mail: BUGEAUD@PARI.U-STRASBG.FR
INSTITUTE OF MATHEMATICS KOSSUTH LAJOS UNIVERSITY 4010 DEBRECEN, HUNGARY E-mail: GYORY@MATH.KLTE.HU

Received on 27.12.1994

and in revised form on 18.4.1995 